\title{
Letter to Editor Articles in Iran: An Index for Critical Paper Reading
}

\author{
Hamidreza Karimi-Sari, ${ }^{1,2}$ and Mohammad Saeid Rezaee-Zavareh ${ }^{1,2,}{ }^{*}$ \\ ${ }^{1}$ Student Research Committee, Baqiyatallah University of Medical Sciences, Tehran, IR Iran \\ ${ }^{2}$ Scientometrics Department of Middle East Liver Diseases (MELD) Center, Tehran, IR Iran \\ Corresponding author: Mohammad Saeid Rezaee-Zavareh, Student Research Committee, Baqiyatallah University of Medical Sciences, Tehran, IR Iran. Tel: +98-2188945188, \\ E-mail: Dr_Rezaee@Live.com
}

Received 2016 December 05; Accepted 2016 December 07.

Keywords: Letter to Editor, Paper, Iran, Turkey, Middle East

Most journals have a section for the letter to editor (LTE) or correspondence, which is a mechanism for proposing criticisms, comments, and questions about published papers. It is considered as a system for the post publication review $(1,2)$. Furthermore, LTEs may even consist of research data or propose a hot topic in a special field. Considering the first definition of LTEs, they can show the readability of a journal and keep it alive by cooperating with the readers (3). We believe that the number of LTEs in each country may be defined as an index, showing the number of real readers in that country. Therefore, we aimed at evaluating the number of LTEs in Iran based on Web of Science, one of the important scientific databases in the world, and to compare it to Turkey, which is the top Middle Eastern country with respect to the number of scientific papers.

We found a total of 3,094 LTEs for Iran compared to 11,041 for Turkey in the last decade. Figure 1 displays the percentage of LTEs among all types of papers in Iran and Turkey from January 2006 to December 2015 according to the Web of Science. We did not limit our search to a specific field of research and our last search was conducted on June 18, 2016. Based on Figure 1, the percent of LTEs for Iran has been considerably lower than that of Turkey for the past decade. Moreover, this trend has recently been decreased for Iran, but Turkey has had a growing trend. Thus, it could be assumed that the number of LTEs depends on the authors' interest and journals' policy in that region. The top three journals that published Iranian LTEs in the past decade have been Iranian red crescent journal of medicine $(\mathrm{N}=150)$, Iranian journal of public health $(\mathrm{N}=126)$, and journal of research in medical sciences $(\mathrm{N}$ = 103). Furthermore, the top three Iranian authors who wrote most LTEs in the past decade were Namazi, Hamid ( $N=70$, Shiraz University of Medical Sciences), Alavian, Seyed Moayed $(\mathrm{N}=66$, Baqiyatallah University of Medical Sciences), and Gharibzadeh, Shahriar ( $\mathrm{N}=61$, Amirkabir University of Technology).

Although type of LTE articles is not just related to post publication review, the difference between Iran and Turkey is indeed considerable, meaning that post publication reviews and critical paper reading occur less among Iranian authors compared to their Turkish counterparts. Several issues can be proposed here including lack of awareness about the importance of this type of article and its low score in the universities' promotion system etc. We suggest that the culture for writing LTEs be promoted among Iranian readers through holding related educational workshops and journal clubs. Furthermore, journals' editors should make appropriate policies to pave the way to accept more of readers' comments and ideas, which could certainly help conducting more reliable and trustable research papers.

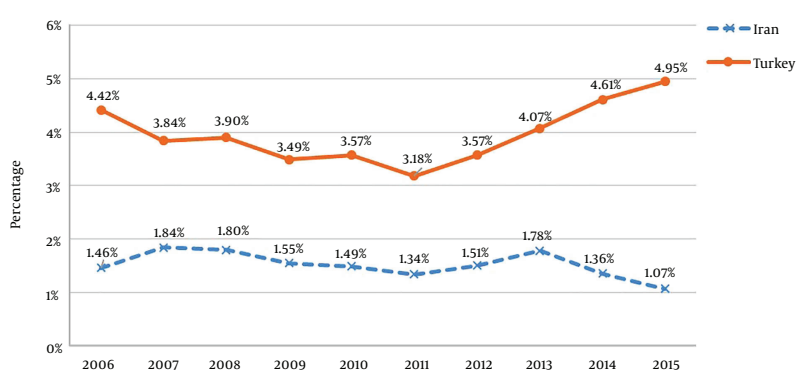

Figure 1. Percentage of the Letter to Editors Among All Types of Papers in Iran and Turkey According to the Web of Science Database (2006 - 2015)

\section{Footnote}

Authors' Contribution: Concept: Mohammad Saeid Rezaee-Zavareh; data acquisition and analysis: Hamidreza Karimi-Sari and Mohammad Saeid Rezaee-Zavareh; drafting the manuscript: Hamidreza Karimi-Sari; critical revision: Mohammad Saeid Rezaee-Zavareh; final approval and agreement: Hamidreza Karimi-Sari and Mohammad Saeid Rezaee-Zavareh. 


\section{References}

1. Florian RV. Aggregating post-publication peer reviews and ratings. Front Comput Neurosci. 2012;6:31. doi: 10.3389/fncom.2012.00031. [PubMed: 22661941].
2. Ghanbari A, Rad SAD. Post publication peer review in Iranian biomedical journals. Galen Med J. 2015;4(1):1-7.

3. Brown CJ. Unvarnished viewpoints and scientific scrutiny. Letter to the editor provide a forum for readers and help make a journal accountable to the medical community. CMAJ. 1997;157(6):792-4. [PubMed: 9333525]. 